\title{
Green/Yellow Solid-State Lighting via Radiative and Nonradiative Energy Transfer Involving Colloidal Semiconductor Nanocrystals
}

\author{
Sedat Nizamoglu, Student Member, IEEE, Emre Sari, Student Member, IEEE, Jong-Hyeob Baek, In-Hwan Lee, \\ and Hilmi Volkan Demir, Member, IEEE
}

\begin{abstract}
LEDs made of $\operatorname{In}_{x} \mathrm{Ga}_{1-x} \mathrm{~N}$ and $\left(\mathrm{Al}_{x} \mathrm{Ga}_{1-x}\right)_{1-y} \operatorname{In}_{y} \mathrm{P}$ suffer from significantly reduced quantum efficiency and luminous efficiency in the green/yellow spectral ranges. To address these problems, we present the design, growth, fabrication, hybridization, and characterization of proofof-concept green/yellow hybrid LEDs that utilize radiative and nonradiative [Förster resonance energy transfer (FRET)] energy transfers in their colloidal semiconductor nanocrystals (NCs) integrated on near-UV LEDs. In our first NC-LED, we realize a color-converted LED that incorporate green-emitting $\mathrm{CdSe} / \mathrm{ZnS}$ core/shell NCs $\left(\lambda_{\mathrm{PL}}=548 \mathrm{~nm}\right)$ on near-UV InGaN/GaN LEDs $\left(\lambda_{E L}=379 \mathrm{~nm}\right)$. In our second NC-LED, we implement a color-converted FRET-enhanced LED. For that, we hybridize a custom-design assembly of cyan- and green-emitting $\mathrm{CdSe} / \mathrm{ZnS}$ core/shell NCs $\left(\lambda_{P L}=490\right.$ and $\left.548 \mathrm{~nm}\right)$ on near-UV LEDs. Using a proper mixture of differently sized NCs, we obtain a quantum efficiency enhancement of $9 \%$ by recycling trapped excitons via FRET. With FRET-NC-LEDs, we show that it is possible to obtain a luminous efficacy of $425 \mathrm{~lm} / \mathrm{W}_{\text {opt }}$ and a luminous efficiency of $94 \mathrm{~lm} / \mathrm{W}$, using near-UV LEDs with a $40 \%$ external quantum efficiency. Finally, we investigate FRET-converted light-emitting structures that use nonradiative energy transfer directly from epitaxial quantum wells to colloidal NCs. These proof-of-concept demonstrations show that FRET-based NC-LEDs hold promise for efficient solid-state lighting in green/yellow.
\end{abstract}

Index Terms-Förster resonance energy transfer (FRET), green/yellow, InGaN/GaN, LEDs, nanocrystals (NCs), nonradiative energy transfer.

Manuscript received December 1, 2008; revised December 24, 2008 and January 20, 2009. Current version published August 5, 2009. This work was supported in part by the Turkish National Academy of Sciences Distinguished Young Scientist Award Program (TUBA GEBIP), in part by the European Science Foundation European Young Investigator Award Program (ESF-EURYI) under Grant EU MOON 02139, and in part by TUBITAK EEEAG under Grant 107E297, Grant 104E114, Grant 106E020, Grant 107E080, Grant 105E065, and Grant 105E066.

S. Nizamoglu and E. Sari are with the Department of Electrical and Electronics Engineering, Nanotechnology Research Center, and the Institute of Materials Science and Nanotechnology, Bilkent University, Ankara 06800, Turkey (e-mail: sedatn@ee.bilkent.edu.tr; emresari@bilkent.edu.tr).

J.-H. Baek is with Korea Photonics Technology Institute, Gwangju 500-460, Korea (e-mail: jhbaek@kopti.re.kr).

I.-H. Lee is with Yale University, New Haven, CT 06511 USA, on leave from the School of Advanced Materials Engineering, Research Center of Industrial Technology, Chonbuk National University, Chonju 561-756, Korea (e-mail: ihlee@chonbuk.ac.kr).

H. V. Demir is with the Department of Electrical and Electronics Engineering, the Department of Physics, Nanotechnology Research Center, and the Institute of Materials Science and Nanotechnology, Bilkent University, Ankara 06800, Turkey (e-mail: volkan@bilkent.edu.tr).

Color versions of one or more of the figures in this paper are available online at http://ieeexplore.ieee.org.

Digital Object Identifier 10.1109/JSTQE.2009.2015680

\section{INTRODUCTION}

$\mathbf{T}$ ODAY solid-state lighting is of great importance due to the financial benefits it offers (e.g., 50\% saving in the global electricity consumption of lighting in principle) and its potential to combat environmental problems (e.g., climate change) [1]. In a recent study, the photometric conditions that achieve ultraefficient solid-state lighting have been investigated [2], [3]. According to this study, a luminous efficacy of optical radiation at $408 \mathrm{~lm} / \mathrm{W}_{\mathrm{opt}}$ and a color rendering index (CRI) of 90 at a correlated color temperature (CCT) of $3000 \mathrm{~K}$ are simultaneously achievable. To obtain these photometric properties, LEDs that emit in blue, green, yellow, and red colors (at 463, 530, 573, and $614 \mathrm{~nm}$ with relative optical power levels of 1/8, 2/8, 2/8, and 3/8, respectively) are required [2], [3].

$\mathrm{In}_{x} \mathrm{Ga}_{1-x} \mathrm{~N}$ material system has the potential to cover the whole visible by changing the In composition $(x)$; however, it has technically been extremely challenging to obtain efficient green and yellow LEDs especially at these wavelengths (i.e., at 530 and $573 \mathrm{~nm}$, respectively) [4]. Though blue-emitting InGaN/GaN LEDs may reach high external quantum efficiency [5], [6], those LEDs made of the same material system begin to suffer from significantly reduced internal quantum efficiency (and thus substantially reduced luminous efficiency) due to the increased In content in multiquantum wells (multiQWs) while approaching green color [7]-[9]. In addition, the resulting optical emission is further reduced due to the increased internal fields in the QWs that separate electron and hole wavefunctions. Furthermore, undesired defects and compositional inhomogeneities also further decrease the efficiency of green LEDs [2].

For red LEDs, it is possible to achieve high quantum efficiencies around $650 \mathrm{~nm}$ by utilizing another material system, a quaternary alloy $\left(\mathrm{Al}_{x} \mathrm{Ga}_{1-x}\right)_{1-y} \operatorname{In}_{y} \mathrm{P}$. By using this quaternary, it is possible to cover from 650 to $580 \mathrm{~nm}$. However, the efficiencies significantly decrease approaching the yellow color. Therefore, there exists a significant gap in the green and yellow spectral regions to make efficient LEDs.

To address these problems, we propose and develop proofof-concept green/yellow hybrid LEDs that use both radiative energy transfer and nonradiative energy transfer (Förster resonance energy transfer (FRET), or fluorescence resonance energy transfer) in their integrated colloidal semiconductor nanocrystals (NCs) for color conversion on near-UV LEDs (Fig. 1). Our experimental demonstrations indicate that the use of FRET 


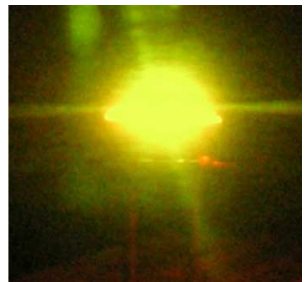

Fig. 1. Exemplary picture of a yellow hybrid LED while emitting at $573 \mathrm{~nm}$.

in color conversion in green and yellow allows for significant enhancement in efficiency. Such FRET-converted LEDs hold great promise for efficient lighting, especially in green and yellow where the existing material systems and LED technologies suffer from low performance.

In conventional color conversion technique, phosphors are commonly used to generate white light. Phosphors provide wideband emission with high quantum efficiency across the visible. It is, however, difficult to tune their optical emission properties and obtain a spectrally narrow emission band (e.g., green or yellow emission alone). As an alternative, though, semiconductor NCs exhibit favorable properties for use in LED applications. They reveal tunable emission using quantum confinement effect [10], [11]. They show spectrally pure, narrow, and symmetric photoluminescence (PL) with large PL quantum yields and high photostability. They exhibit increasing absorption levels toward shorter wavelengths, relatively high photobleaching thresholds, and small spectral overlap between absorption and emission spectra [12]. Furthermore, various film deposition techniques including Langmuir-Blodgett, spin casting, and electrostatic layer-by-layer film deposition can be conveniently used to make good quality film. Therefore, these quasi-zerodimensional semiconductor materials have been both theoretically and experimentally widely investigated [12]-[18]. Because of the tunable nature of their optical emission, they have also been exploited in various LED applications especially for hybrid white LEDs [19]-[24]. Unlike these previous studies of our group and the others, here we demonstrate hybrid LEDs that also employ FRET as a nonradiative means of energy transfer in color-converting colloidal NCs for increased efficiency in green/yellow spectral ranges for the first time.

In this paper, we present the design, growth, fabrication, integration, and characterization of NC-based LEDs that utilize direct and FRET-enhanced color conversion. Furthermore, we also investigate FRET-converted light-emitting structures. In our first NC-LED, we use green-emitting $\mathrm{CdSe} / \mathrm{ZnS}$ core/shell NCs $\left(\lambda_{\mathrm{PL}}=548 \mathrm{~nm}\right)$ on near-UV InGaN/GaN LEDs $\left(\lambda_{\mathrm{EL}}=\right.$ $379 \mathrm{~nm}$ ). In our second NC-LED that additionally employs FRET in NCs, we use a custom-design assembly of cyanand green-emitting CdSe/ZnS core/shell NCs $\left(\lambda_{\mathrm{PL}}=490\right.$ and $548 \mathrm{~nm}$, respectively) to recycle the trapped excitons into NCs via FRET. In this proof-of-concept FRET-enhanced NC-LED, we achieve a quantum efficiency enhancement of $9 \%$ with respect to the first NC-LED. Finally, we examine FRET directly from epitaxial QWs to colloidal NCs in their close proximity.

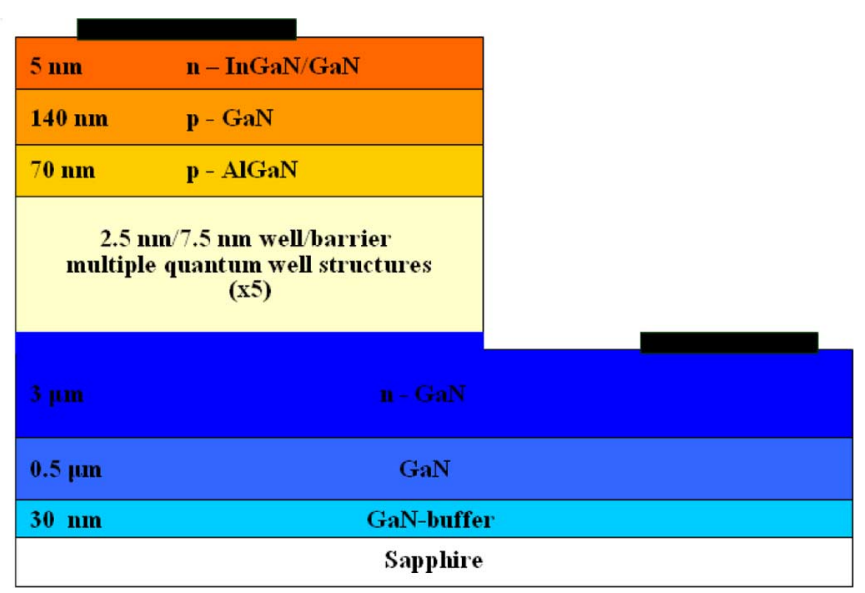

Fig. 2. Epitaxial design of our near-UV InGaN/GaN LED.

Using FRET pumping in color conversion, a potential efficiency enhancement is predicted in such hybrid LEDs.

\section{NEAR-UV LED}

For the growth of our near-UV LED, we employ a GaN dedicated metal-organic chemical vapor deposition (MOCVD) system and use a p-i-n epitaxial design for the near-UV LED, as shown in Fig. 2. At first, we begin with a 30-nm-thick GaN buffer layer and continue with a $0.5-\mu \mathrm{m}$-thick GaN layer to increase the crystal quality of the device epitaxial layers. Subsequently, we grow a 3- $\mu \mathrm{m}$-thick Si-doped n-type contact layer. We continue with the epitaxial growth of $2.5 \mathrm{~nm} / 7.5 \mathrm{~nm} \mathrm{InGaN} / \mathrm{GaN}$ $\mathrm{QW} /$ barrier structure. We repeat the well/barrier structure five times for multi-QW region. Finally, we finish our growth with layers that consist of Mg-doped 70-nm-thick AlGaN, 140-nmthick Mg-doped GaN layer, and 2.5-nm-/2.5-m-thick Si-doped InGaN/GaN layers. In the device fabrication, we use standard semiconductor processing including photolithography, thermal evaporator (metallization), reactive ion etch (RIE), and rapid thermal annealing. Finally, we make wire bonds to the device contacts in its package.

Our near-UV LED has a turn-ON voltage approximately at $3 \mathrm{~V}$ and an electroluminescence (EL) peak wavelength at $379 \mathrm{~nm}$, as shown in the inset of Fig. 3. Our near-UV LED exhibits an external quantum efficiency of around $8 \%$ at low current levels. For example, the optical power is $5.8 \mathrm{~mW}$ at $19.05 \mathrm{~mA}$. However, we observe a strong rollover in the efficiency with increasing input current, as our packaged LED does not include a heat sink and its full package is not designed for proper heat management. With a decreased level of device efficiency, the near-UV LED reaches an output optical power level of $8.5 \mathrm{~mW}$ at $43 \mathrm{~mA}$, as shown in Fig. 3.

\section{COLOR-CONVERTED LED}

For the first NC-LED, we use green-emitting $\mathrm{CdSe} / \mathrm{ZnS}$ core/shell NCs as the color converters. Our green-emitting NCs have a concentration of $70 \mathrm{nmol} / \mathrm{mL}$ in toluene and their diameters are around $3.4 \mathrm{~nm}$ with a size dispersion of $<5 \%$. Their molecular weight is $100 \mu \mathrm{g} / \mathrm{nmol}$ and they exhibit an in-solution 


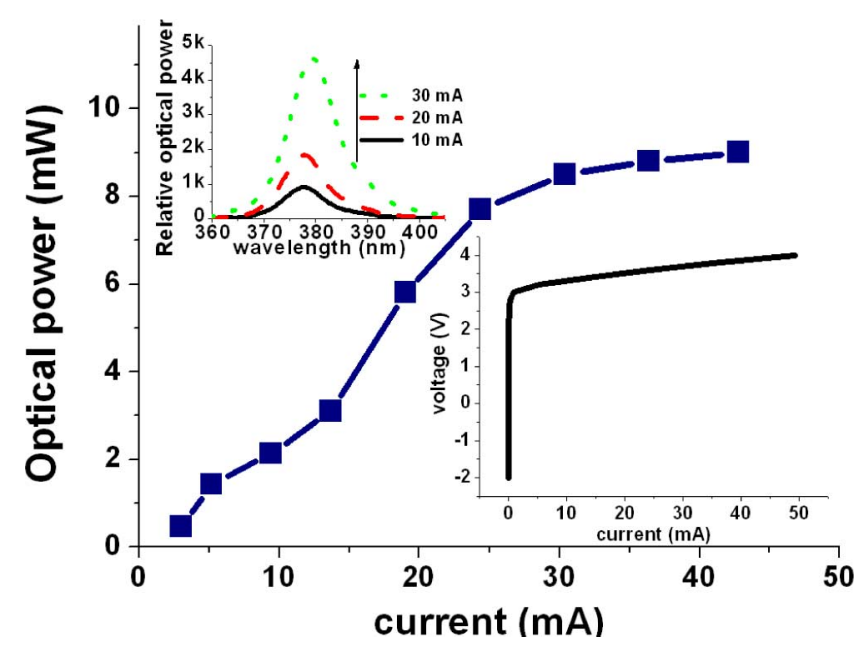

Fig. 3. $L-I$ of our near-UV LED measured in an integrating sphere at room temperature (with no heat sink or active cooling) along with its EL spectra and $I-V$ given in the insets.

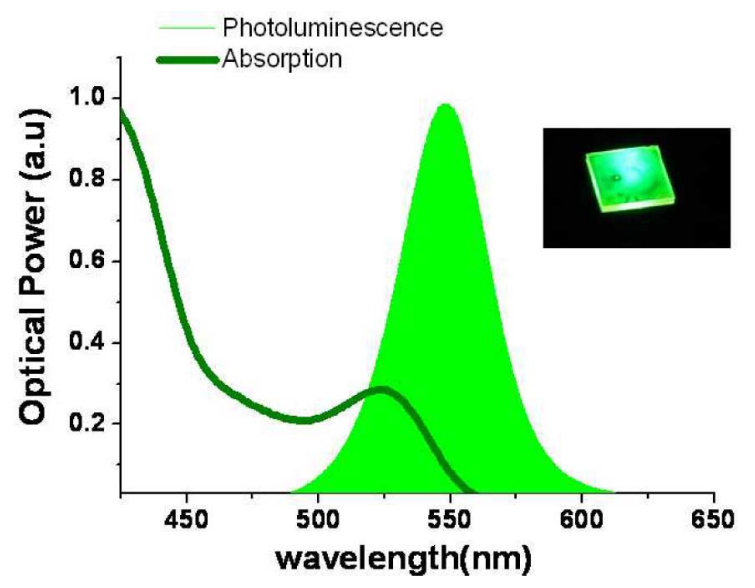

Fig. 4. Emission and absorption spectra of green-emitting CdSe/ZnS core/shell NCs along with their PL picture given in the inset.

quantum efficiency of $>50 \%$. Their emission and absorption spectra are shown in Fig. 4. Toward shorter wavelengths, their optical absorption increases rapidly.

For hybrid light generation, we integrate closely packed NCs on a near-UV LED. The operating principle for such NC-LEDs relies on the optical excitation of the integrated NC films by the electrically driven near-UV LED. As a result of optical pumping, the NC PL generates the desired green/yellow light.

For our first NC-LED, we integrate a carefully determined amount of $9.1 \mathrm{nmol}$ green-emitting CdSe/ZnS NCs on our LED. By using this exact amount of NCs, all of the LED EL at $379 \mathrm{~nm}$ is fully down-converted to NC emission by the NC luminophors. In Fig. 5, as the current injection level increases, the optical output power of our LED expectedly increases as well. When the current level reaches $19.05 \mathrm{~mA}$, the optical power becomes $1.81 \mathrm{~mW}$, which corresponds to a quantum efficiency of $46 \%$ for the integrated NC layer. The resultant luminescence of the hybrid LED is shown as a function of increasing current injection level in the inset of Fig. 5. Here, it is important to note that the green $\mathrm{NC}$ emission shifted $20 \mathrm{~nm}$ from 548 to $568 \mathrm{~nm}$ in

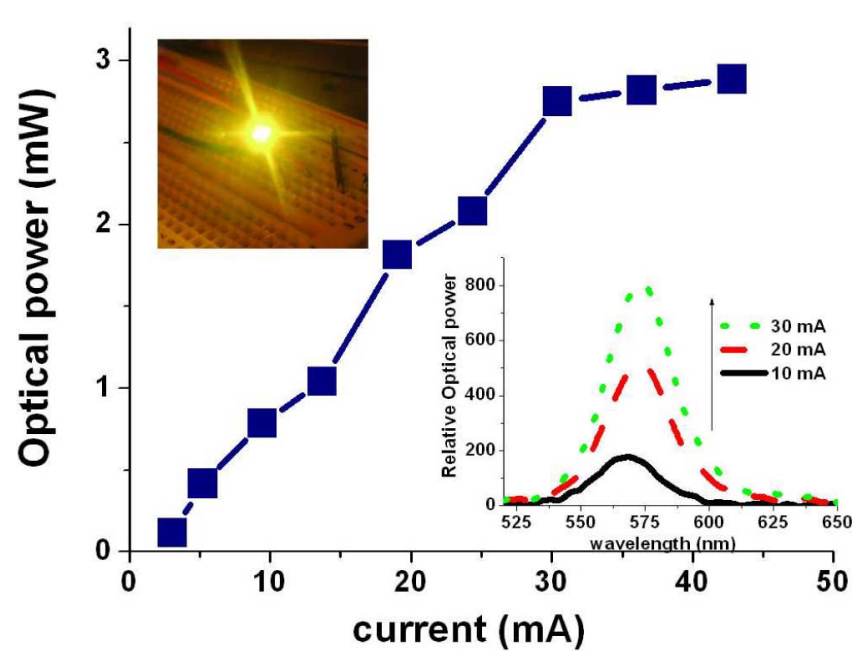

Fig. 5. Total optical power of green NC $\left(\lambda_{\mathrm{PL}}=548 \mathrm{~nm}\right)$ hybridized on near$\mathrm{UV} \operatorname{LED}\left(\lambda_{\mathrm{EL}}=379 \mathrm{~nm}\right)$ at different levels of current injection at room temperature (with no heat sink or active cooling), along with the emission spectra and picture of the hybrid NC-LED when electrically driven.

TABLE I

PHOTOMETRIC PROPERTIES OF OUR COLOR-CONVERTED NC-LED AT VARIOUS CURRENT INJECTION LEVELS

\begin{tabular}{|c|c|c|c|}
\hline $\mathbf{I}(\mathbf{m A})$ & $\mathbf{X}$ & $\mathbf{Y}$ & $\mathbf{C C T}(\mathbf{K})$ \\
\hline $\mathbf{9 . 4 5}$ & 0.42 & 0.54 & 4008 \\
\hline $\mathbf{1 9 . 5 0}$ & 0.45 & 0.50 & 3423 \\
\hline $\mathbf{3 0 . 4 0}$ & 0.46 & 0.51 & 3260 \\
\hline
\end{tabular}

the solid film. This is mainly due to the nonradiative energy transfer among NCs because of their size distribution $(<5 \%)$. This size variance leads to slightly modified effective bandgaps. Consequently, the absorption of the relatively small-sized NCs and emission of the relatively large-sized NCs overlap, which enables energy transfer among them. However, this is not a fundamental problem. Since the starting NC peak emission can be conveniently set at any desired wavelength across the visible, this redshift can be easily compensated for using NCs with a shorter starting peak emission wavelength.

As the current injection level increases, the emission of packaged hybrid LED redshifts due to thermal effects that pushes our operating wavelength closer to $573 \mathrm{~nm}$ for yellow LED in ultraefficient solid-state lighting. Thus, $(x, y)$ tristimulus coordinates of the hybrid LED moves from $(0.42,0.54)$ to $(0.46,0.51)$ and its CCT shifts from 4008 to $3260 \mathrm{~K}$, as summarized in Table I. We also measure the luminous efficacy of optical radiation and luminous efficiency of the source using (1) and (2), where $v(\lambda)$ is the eye sensitivity function and $P(\lambda)$ is the power spectral density (i.e., the light power emitted per unit wavelength) [6]. At $19.05 \mathrm{~mA}$, our color-converted LED exhibits a luminous efficacy of $425 \mathrm{~lm} / \mathrm{W}_{\text {opt }}$ and a luminous efficiency of $17 \mathrm{~lm} / \mathrm{W}$

$$
\begin{aligned}
\mathrm{LE}_{\text {Optical }} & =\left(683 \frac{\mathrm{lm}}{\mathrm{W}}\right) \frac{\int P_{\text {optical }}(\lambda) v(\lambda) d \lambda}{\int P_{\text {optical }}(\lambda) d \lambda} \\
\mathrm{LE}_{\text {Electrical }} & =\left(683 \frac{\mathrm{lm}}{\mathrm{W}}\right) \frac{\int P_{\text {optical }}(\lambda) v(\lambda) d \lambda}{V I} .
\end{aligned}
$$




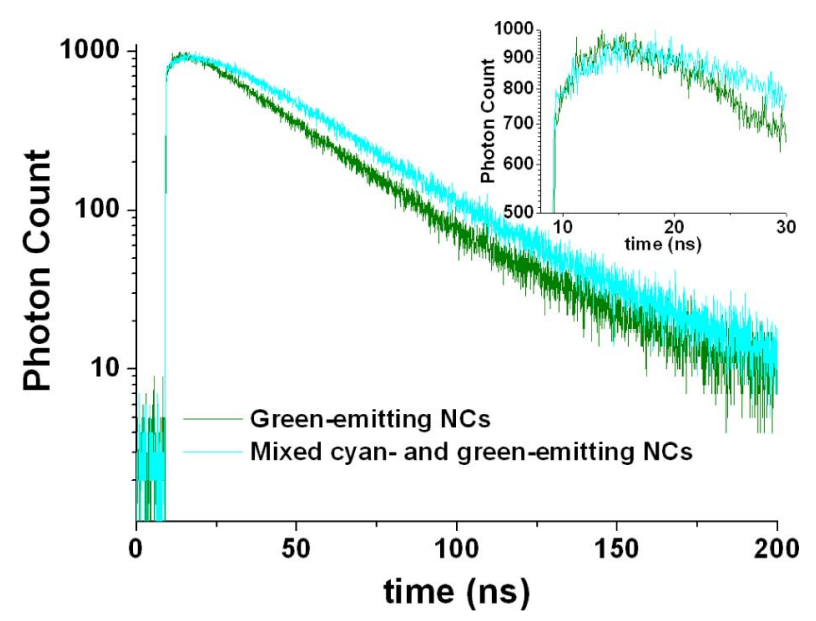

Fig. 6. Time-resolved spectroscopy measurements of the integrated greenemitting CdSe $/ \mathrm{ZnS}$ core/shell NCs $\left(\lambda_{\mathrm{PL}}=548 \mathrm{~nm}\right)$ and mixed cyan- and green-emitting CdSe $/ Z n S$ core/shell NCs $\left(\lambda_{\mathrm{PL}}=490\right.$ and $548 \mathrm{~nm}$, respectively) on near-UV LED $\left(\lambda_{\mathrm{EL}}=379 \mathrm{~nm}\right)$.

Fig. 6 shows the time-resolved spectroscopy measurements of the integrated NC layers at $556 \mathrm{~nm}$. In Fig. 6, the energy rise (i.e., exponential increase) observed at $9 \mathrm{~ns}$ comes from the energy transfer. For these time-resolved spectroscopy measurements, we use a FluoTime 200 spectrometer (PicoQuant) with a timecorrelated single photon counting (TCSPC) system of PicoHarp 300 that has a calibrated minimum possible time resolution of 4 ps. To pump NC solids, we use a laser head at an emission wavelength of $375 \mathrm{~nm}$ with light pulses as short as $70 \mathrm{ps}$ and a photon multiplier tube (PMT) as the detector. Using an output monochromator, we characterize all of the prepared NC solids at $556 \mathrm{~nm}$, corresponding to the peak emission wavelengths of the NCs. For the data analysis, we use the software FluoFit to take into account the instrumental response function (IRF).

To further analyze the PL decay curves of our NCs, we make fits to experimentally measured decay curves in Fig. 6 using a multiexponential model fit and convoluting with the laser diode response function at $375 \mathrm{~nm}$. The resultant fitting parameters are summarized in Table II. Here, for our decays, we have two distinct lifetime components. The first lifetime component (associated with $\tau_{1}$ ) is the general lifetime of the NCs, which is expectedly on the order of tens of nanoseconds [12], [13]. The second lifetime component (associated with $\tau_{2}$ ) is an energy increase, which we conclusively differentiate with its negative amplitude. Here, it is worth noting that the distinct negative amplitude stems only possibly from the nonradiative energy transfer among NCs due to their size distribution, as discussed previously. For comparison, the cases of mixed green- and cyanemitting NCs that exhibit intentionally strong energy transfer in Fig. 6 (and also in Table II) will be discussed in the next section.

\section{COLOR-CONVERTED FRET-ENHANCED LED}

To enhance the efficiency of the hybrid LED, we integrate a carefully designed assembly of $3.4 \mathrm{nmol}$ cyan-emitting NCs and $4.9 \mathrm{nmol}$ green-emitting NCs as an energy gradient structure on a similar near-UV LED, which increases the overall quantum effi-
TABLE II

MultieXPonential Fitting PaRAmeters For Transient PL DeCAys IN FIG. 6

\begin{tabular}{|c|c|c|c|c|}
\hline & $\mathbf{A}_{\mathbf{1}}$ & $\boldsymbol{\tau}_{\mathbf{1}}(\mathbf{n s})$ & $\mathbf{A}_{\mathbf{2}}$ & $\boldsymbol{\tau}_{\mathbf{2}}(\mathbf{n s})$ \\
\hline \hline $\begin{array}{c}\text { Only green-emitting } \\
\text { NCs }\end{array}$ & 219.69 & 32.78 & -99.70 & 3.18 \\
\hline $\begin{array}{c}\text { Mixed cyan- and } \\
\text { green-emitting NCs }\end{array}$ & 309.85 & 32.78 & -166.77 & 12.10 \\
\hline
\end{tabular}

$A_{n}$ and $\tau_{n}$ are the associated amplitudes and decay time constants of multiexponential decay fits to the experimental PL decay curves; $n=1,2$.

ciency of the integrated NC layer. Here, the physical mechanism of the quantum efficiency enhancement stems from the ability to transfer excitation energy of the trapped excitons captured in defect states of donor NCs into acceptor NCs using nonradiative resonance energy transfer, as discussed in [25] and [26]. With the optical excitation by incoming near-UV light, the photogenerated electron and hole pairs relax rapidly (in hundreds of femtoseconds) to their respective conduction and valence bands, some of which subsequently make interband transitions for radiative recombination. However, some of these photogenerated electron-hole pairs get trapped in the defect states, typically leading to nonradiative recombination. Using nonradiative energy transfer, some portions of these trapped excitons are transferred to the green-emitting NCs that serve as acceptors for these defect states, a part of which, in turn, contribute to PL of these acceptors. Thus, the overall luminescence increases because of the enhanced emission of the acceptors by effectively recycling the trapped excitons (and thus otherwise lost excitation energy) in the defect states of donors.

In Fig. 6, the time-resolved spectroscopy measurements of mixed cyan- and green-emitting NC film are presented. We observe in Fig. 6 (and also in Table II) that the energy rise (i.e., the exponential increase) in the energy gradient structure of mixed cyan-green NCs is more dominant with respect to the case of only green NCs, because of the strong exciton migration to green-emitting NCs. By using the physical process of FRET, we obtain the resulting luminescence of the hybrid device, as shown in Fig. 7. Here, the quantum efficiency of the NC film is enhanced by $9 \%$.

It is important to note that cyan-emitting NCs are fully quenched by transferring their energy to green-emitting NCs. As a result of the enhanced quantum efficiency of the mixed NC assembly, at $19.05 \mathrm{~mA}$, the FRET-enhanced NC-LED reaches a luminous efficiency of $19 \mathrm{~lm} / \mathrm{W}$, while the luminous efficacy of optical radiation is maintained at $425 \mathrm{~lm} / \mathrm{W}_{\mathrm{opt}}$. This FRETNC-LED has its chromaticity coordinates at $(x, y)=(0.46$, 0.51 ). As the current injection level increases, the emission of FRET-NC-LED makes expectedly a redshift from 568 to $573 \mathrm{~nm}$ due to the additional heating. Its tristimulus coordinates and CCT correspondingly shift from $(0.40,0.49)$ to $(0.45,0.52)$ and from 4077 to $3441 \mathrm{~K}$, respectively, as given in Table III. In our FRET-NC-LED, NCs reach a quantum efficiency level as high as $55 \%$. By using a state-of-the-art near-UV LED with an external quantum efficiency of $40 \%$, this implies that it is possible to obtain color-converted FRET-enhanced LEDs with an expected external quantum efficiency of $22 \%$ and a predicted luminous efficiency of $94 \mathrm{~lm} / \mathrm{W}$. 


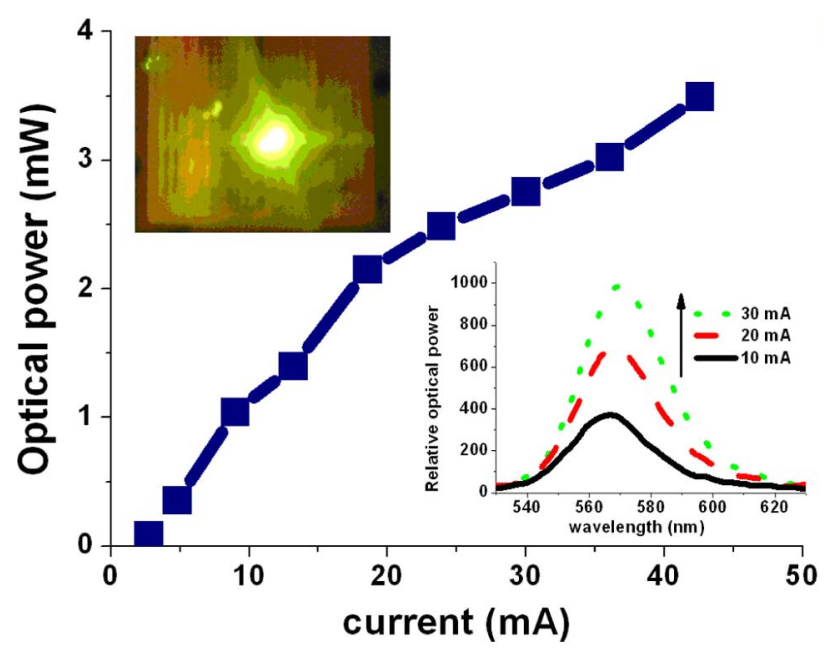

Fig. 7. Total optical power of mixed cyan- and green-emitting NCs $\left(\lambda_{\mathrm{PL}}=\right.$ 490 and $548 \mathrm{~nm}$, respectively) hybridized on near-UV LED $\left(\lambda_{\mathrm{EL}}=379 \mathrm{~nm}\right)$ at different levels of current injection at room temperature (with no heat sink or active cooling), along with the emission spectra and picture of the resulting FRET-NC-LED when electrically driven.

TABLE III

PHOTOMETRIC PROPERTIES OF OUR COLOR-CONVERTED FRET-ENHANCED NC-LED AT VARIOUS CURRENT INJECTION LEVELS

\begin{tabular}{|c|c|c|c|}
\hline $\mathbf{I}(\mathbf{m A})$ & $\mathbf{x}$ & $\mathbf{Y}$ & $\mathbf{C C T}(\mathbf{K})$ \\
\hline $\mathbf{9 . 4 5}$ & 0.40 & 0.49 & 4077 \\
\hline $\mathbf{1 9 . 5 0}$ & 0.44 & 0.51 & 3642 \\
\hline $\mathbf{3 0 . 4 0}$ & 0.45 & 0.52 & 3441 \\
\hline
\end{tabular}

\section{FRET-CONVERTED Light-EMitTing STRUCTURES}

In the first NC-LED, color conversion is achieved through only optical pumping (i.e., radiative energy transfer) that involves two recombination processes. The first radiative recombination process occurs in the electrically driven QWs of the LED, and subsequently, their emitted photons excite the integrated NC luminophors that further luminescence via a second radiative recombination process. On the other hand, rather than using two-step recombination processes, color conversion using only one recombination step is also possible via nonradiative energy transfer directly from epitaxial QWs to colloidal NCs [which are chemically synthesized quantum dots (QDs)] in their close proximity [13]. The advantage of such energytransferring hybrid structure is that recombination takes place directly in the NCs (i.e., after QWs transfer their excitation energy to these QDs for the recombination process to directly take place in the dots) for color conversion. Therefore, using nonradiative energy transfer for color conversion facilitates a direct excitation of NCs with a more efficient, single-step process, as opposed to merely optical pumping that requires two steps of radiative recombination.

To nonradiatively pump the NCs by using FRET, we use a special epiwafer design, as shown in Fig. 8. In its epitaxial growth, we start with a 30-nm-thick GaN buffer layer on a double-side-polished c-plane sapphire and a $2-\mu \mathrm{m}$-thick GaN buffer layer to increase the crystal quality of the device epitaxial layers. Subsequently, we grow a 1.2- $\mu \mathrm{m}$-thick Mg-doped p-type

\begin{tabular}{|lc|}
\hline $2 \mathrm{~mm}$ & GaN cap layer \\
\hline $3 \mathrm{~nm}$ & InGaN SQW \\
\hline $50 \mathrm{~mm}$ & p - GaN \\
\hline $1.2 \mu \mathrm{m}$ & p - GaN \\
\hline $2 \mu \mathrm{m}$ & undoped GaN \\
\hline $30 \mathrm{~mm}$ & GaN buffer \\
\hline & Sapphire \\
\hline
\end{tabular}

Fig. 8. Epitaxial structure of our wafer for energy transfer from single QW (SQW) to colloidal NCs for yellow/green emission.

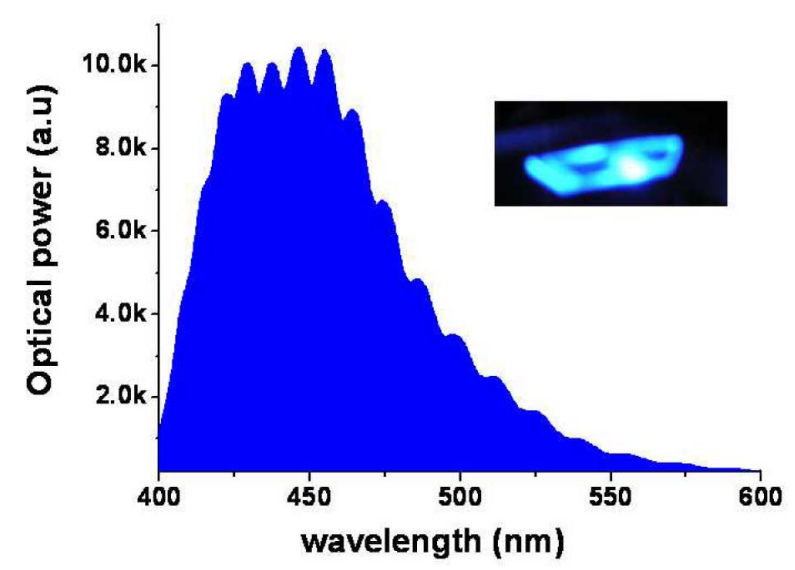

Fig. 9. PL of our epiwafer for energy transfer from SQW to colloidal NCs.

$\mathrm{GaN}$ layer and a 50-nm-thick highly doped p-type GaN layer. We then continue the epi-growth with a 3 -nm-thick $\mathrm{InGaN}$ well. Finally, we finish our growth with a 2-nm cap layer that enables the dipole-dipole interaction of the QW and NCs. For steadystate measurements, we use a Jobin Yvon Triax 500 CCD PL system with an $\mathrm{He} / \mathrm{Cd}$ laser at $325 \mathrm{~nm}$, and the PL of the resulting wafer is shown in Fig. 9. The resultant wavy PL behavior stems for phase separation of the QW. Here, since the optical absorption of the NCs increases toward shorter wavelengths (as shown in the inset of Fig. 4), the overlap of the QW emission and the NC absorption makes strong dipole-dipole interaction between these NCs and QW possible.

In this color-converting hybrid structure, the energy transfer is possible as a result of the dipole-dipole interaction between the QW and NCs, rather than the delocalized behavior of excitons or free carriers, because $\mathrm{ZnS}$ barriers of our NCs prevent tunneling and provide full electronic isolation. We prepare our hybrid structure by integrating NCs on top of the epiwafer and its negative control group by integrating the same density and amount of NCs on quartz substrate. To explore the energy transfer dynamics, we investigate the time-resolved fluorescence of our hybrid system at $556 \mathrm{~nm}$ to observe the energy feeding into NCs, instead of studying the quenching of the QWs. This is because the quenching can also occur because of environmental trap states, but the energy feeding in NC solids can occur merely as a result of the energy transfer to them. 


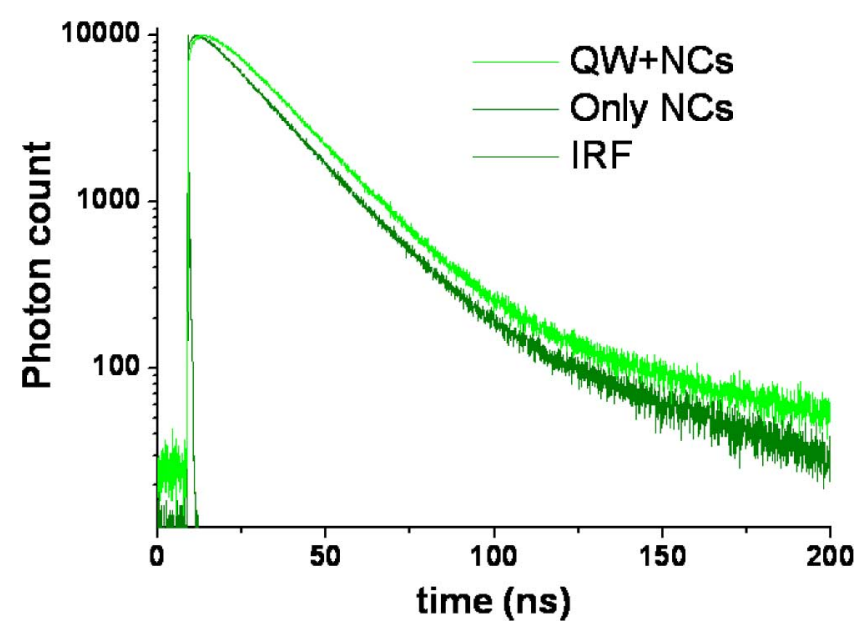

Fig. 10. Time resolved spectroscopy measurements of our hybrid FRETconverted system that consists of both $\mathrm{QW}$ and $\mathrm{NCs}(\mathrm{QW}+\mathrm{NCs})$, compared with that of the negative control group that contains only NCs (only NCs), along with the instrument response function (IRF) of the laser diode emitting at $375 \mathrm{~nm}$, using a TCSPC system of PicoHarp 300 with a time resolution of $64 \mathrm{ps}$.

TABLE IV

MultieXPonential FitTing PaRAmeters For TRANSIENT PL DeCAYS IN FIG. 10

\begin{tabular}{|c|c|c|c|c|c|c|}
\hline & $A_{1}$ & $\tau_{1}(\mathrm{~ns})$ & $\mathbf{A}_{2}$ & $\tau_{2}(\mathrm{~ns})$ & $\mathbf{A}_{3}$ & $\tau_{3}(\mathrm{~ns})$ \\
\hline $\begin{array}{l}\text { Only green- } \\
\text { emitting NCs }\end{array}$ & 14339 & 18.34 & 351 & 70.44 & -4244 & 4.36 \\
\hline $\begin{array}{l}\text { QWs+ green- } \\
\text { emitting NCs }\end{array}$ & 21651 & 18.34 & 695 & 61.43 & -13796 & 7.29 \\
\hline
\end{tabular}

Fig. 10 shows the time-resolved spectroscopy of our hybrid system and that of only the NC solids as the control group. Table IV also summarizes the corresponding multiexponential fitting parameters for these transient PL decays. Here, the first lifetime component (associated with $\tau_{1}$ ) is again the general lifetime of $\mathrm{NC}$ emitters on the order of tens of nanoseconds. The second lifetime component (associated with $\tau_{2}$ ) is relatively weak in amplitude and is attributed to the environmental effects. The third lifetime component (associated with $\tau_{3}$ ) distinctively exhibits negative amplitude, indicating nonradiative energy transfer. In the hybrid FRET-converted structure, this third lifetime component is increased, leading to emission decaying further slower. In agreement with this observation, the transient PL in Fig. 10 also shows a smoother turnaround from the rising edge to the decaying tail (from 8 to $10 \mathrm{~ns}$ ) owing to the energy transfer feeding component of the resulting PL.

In Fig. 11, the steady-state PL spectra of our hybrid FRETconverted system that contains NCs integrated on QW and that of its negative control group that includes only the NCs are depicted. Here, the emission of the NCs in the hybrid system is increased with respect to the case of only NCs as a result of the energy transfer from QW to NCs. Furthermore, in the hybrid system, the emission of NCs is spectrally disturbed, resulting in an asymmetric spectral emission profile, which is another evidence of the nonradiative energy transfer from the QW to NCs.

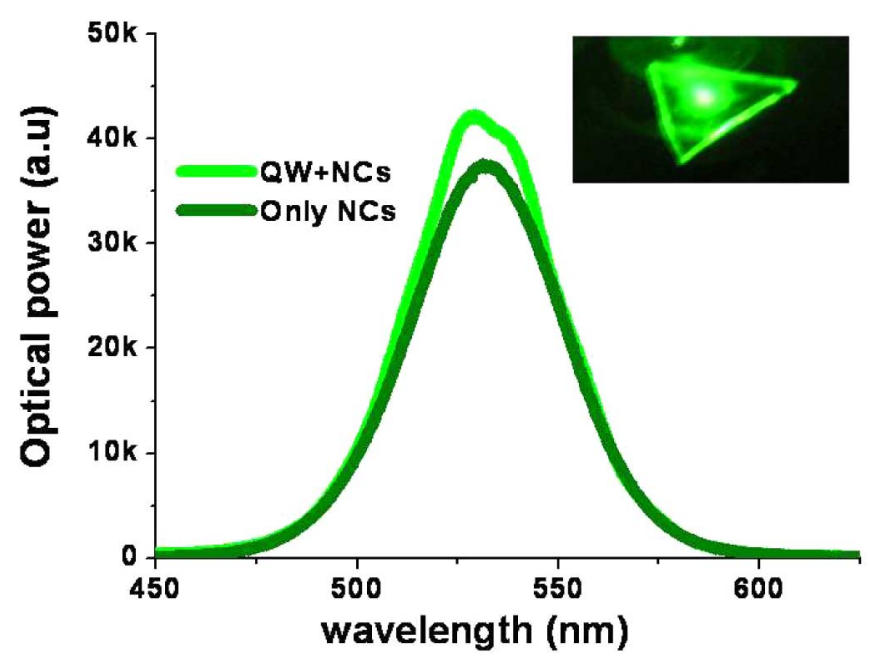

Fig. 11. Steady-state emission spectrum of our hybrid FRET-converted system (QW + NCs) improved by using nonradiative energy transfer pumping of green-emitting $\mathrm{CdSe} / \mathrm{ZnS}$ core/shell $\mathrm{NCs}\left(\lambda_{\mathrm{PL}}=548 \mathrm{~nm}\right)$ by blue-emitting $\mathrm{InGaN} / \mathrm{GaN} \mathrm{QW}\left(\lambda_{\mathrm{PL}}=450 \mathrm{~nm}\right)$ with respect to the case of only NCs.

Nonradiative energy transfer pumping from QWs to NCs for color conversion allows for direct excitation of the NC luminophors, leading to a subsequent single-step radiative recombination process, compared to only radiative energy transfer pumping that requires two subsequent steps of radiative recombination. Using nonradiative energy transfer in addition to radiative transfer, it is possible to make color-converted FRETenhanced LEDs with an external quantum efficiency of $22 \%$, starting with a state-of-the-art near-UV LED that has an external quantum efficiency of $40 \%$. In the case of using only nonradiative energy transfer pumping, though, it is predicted to possibly improve the overall efficiency even further. These preliminary results on FRET conversion implies that such NCbased FRET-converted solid-state lighting holds great promise for future lighting applications.

\section{CONCLUSION}

In this study, we presented the design, growth, fabrication, hybridization, and characterization of hybrid LEDs that integrate colloidal semiconductor NCs as color converters on near-UV InGaN/GaN LEDs. In our proof-of-concept color-converted NC-LED by hybridizing green-emitting $\mathrm{CdSe} / \mathrm{ZnS}$ core/shell NCs, we achieved the luminous efficacy of optical radiation to be $425 \mathrm{~lm} / \mathrm{W}_{\text {opt }}$ and the luminous efficiency to be $17 \mathrm{~lm} / \mathrm{W}$ at a current injection level of $19.05 \mathrm{~mA}$.

In our proof-of-concept color-converted FRET-enhanced NCLED by hybridizing a custom-design assembly of cyan- and green-emitting $\mathrm{CdSe} / \mathrm{ZnS}$ core/shell NCs to facilitate nonradiative energy transfer among them, we demonstrated a quantum efficiency enhancement of $9 \%$ with respect to the colorconverted NC-LED by recycling the trapped excitons into NCs via FRET. At a current injection level of $19.05 \mathrm{~mA}$ while keeping the luminous efficacy of optical radiation at $425 \mathrm{~lm} / \mathrm{W}_{\text {opt }}$, we further improved our hybrid NC-LED to reach a luminous efficiency of $19 \mathrm{~lm} / \mathrm{W}$. In our FRET-NC-LED, NCs reach a quantum 
efficiency level of 55\%. Starting with a $40 \%$ efficiency near-UV LED, a luminous efficiency of $94 \mathrm{~lm} / \mathrm{W}$ and an external quantum efficiency of $22 \%$ are predicted in yellow/green spectral ranges.

Finally, investigating FRET-converted light-emitting structures for direct excitation from epitaxial QWs to colloidal NCs, a further potential efficiency enhancement is predicted for such hybrid LEDs. Currently, experiments on the quantification of FRET conversion enhancement are underway. These proof-ofprinciple demonstrations show that these hybrid NC-LEDs are potentially strong candidates for future lighting applications in green/yellow.

\section{REFERENCES}

[1] The Promise of Solid State Lighting for General Illumination Light Emitting Diodes (LEDs) and Organic Light Emitting Diodes (OLEDs), Optoelectronics Industry Development Association, Washington, DC. (2009). [Online]. Available: http://www.netl.doe.gov/ssl/PDFs/oida_ledoled_rpt.pdf

[2] J. M. Phillips, M. E. Coltrin, M. H. Crawford, A. J. Fischer, M. R. Krames, R. Mueller-Mach, G. O. Mueller, Y. Ohno, L. E. S. Rohwer, J. A. Simmons, and J. Y. Tsao, "Research challenges to ultra-efficient inorganic solid-state lighting," Laser Photon. Rev., vol. 1, no. 4, pp. 307-333, 2007.

[3] J. Y. Tsao, "Challenges to ultra-efficient solid state lighting," in Proc. 21st Annu. Meeting IEEE Lasers Electro-Opt. Soc. Conf., 2008, p. 63, Paper WU4.

[4] M. R. Krames, O. B. Shchekin, R. Mueller-Mach, G. O. Mueller-Mach, Z. Ling, G. Harbers, and M. G. Craford, "Status and future of high-power light-emitting diodes for solid-state lighting," J. Disp. Technol., vol. 3, no. 2, pp. 160-175, Jun. 2007.

[5] S. Nakamura and G. Fasol, The Blue Laser Diode. Berlin, Germany: Springer-Verlag, 1997.

[6] E. F. Schubert, Light-Emitting Diodes. New York: Cambridge Univ. Press, 2006.

[7] I. K. Park, M. K. Kwon, J. O. Kim, S. B. Seo, J. Y. Kim, J. H. Lim, and S. J. Park, "Green light-emitting diodes with self-assembled In-rich InGaN quantum dots," Appl. Phys. Lett., vol. 91, pp. 133105-1-133105-3, 2007.

[8] Y. H. Cho, S. K. Lee, H. S. Kwack, J. Y. Kim, K. S. Lim, H. M. Kim, T. W. Kang, S. N. Lee, M. S. Seon, O. H. Nam, and Y. J. Park, "Carrier loss and luminescence degradation in green-light-emitting InGaN quantum wells with micron-scale indium clusters," Appl. Phys. Lett., vol. 83, pp. 2578-2580, 2003.

[9] C. Bayram, F. H. Teherani, D. J. Rogers, and M. Razeghi, "A hybrid green light emitting diode comprised of $\mathrm{n}-\mathrm{ZnO} /(\mathrm{InGaN} / \mathrm{GaN})$ multi-quantumwells/p-GaN," Appl. Phys. Lett., vol. 93, pp. 081111-1-081111-3, 2008.

[10] A. P. Alivisatos, "Semiconductor clusters, nanocrystals, and quantum dots," Science, vol. 271, no. 5251, pp. 933-937, 1996.

[11] S. Gaponenko, Optical Properties of Semiconductor Nanocrystals. New York: Cambridge Univ. Press, 1998.

[12] V. I. Klimov, A. A. Mikhailovsky, S. Xu, A. Malko, J. A. Hollingsworth, C. A. Leatherdale, H. J. Eisler, and M. G. Bawendi, "Optical gain and stimulated emission in nanocrystal quantum dots," Science, vol. 290, pp. 314-317, 2000.

[13] M. Achermann, M. A. Petruska, S. Kos, D. L. Smith, D. D. Koleske, and V. I. Klimov, "Energy- transfer pumping of semiconductor nanocrystals using an epitaxial quantum well," Nature, vol. 429, pp. 642-646, 2004.

[14] A. L. Rogach, A. Eychmüller, S. G. Hickey, and S. V. Kershaw, "Infrared emitting colloidal nanocrystals: Synthesis, assembly, spectroscopy, and applications," Small, vol. 3, pp. 536-557, 2007.

[15] J. H. Ahn, C. Bertoni, S. Dunn, C. Wang, D. V. Talapin, N. Gaponik, A. Eychmueller, Y. Hua, M. R. Bryce, and M. C. Petty, "White organic light emitting devices incorporating nanoparticles of II-VI semiconductors," Nanotechnology, vol. 18, pp. 335202-1-335202-7, 2007.

[16] I. M. Soganci, S. Nizamoglu, E. Mutlugun, O. Akin, and H. V. Demir, "Localized plasmon-engineered spontaneous emission of $\mathrm{CdSe} / \mathrm{ZnS}$ nanocrystals closely-packed in the proximity of Ag nanoisland films for controlling emission linewidth, peak, and intensity," Opt. Exp., vol. 15, pp. 14289-14298, 2007.

[17] E. Mutlugun, I. M. Soganci, and H. V. Demir, "Nanocrystal hybridized scintillators for enhanced detection and imaging on Si platforms in UV," Opt. Exp., vol. 15, pp. 1128-1134, 2007.
[18] S. Nizamoglu and H. V. Demir, "Nanocrystal based hybrid white light generation with tunable color parameters," J. Opt. A: Pure Appl. Opt., vol. 9, no. 9, pp. S419-S424, 2007.

[19] S. Nizamoglu, G. Zengin, and H. V. Demir, "Color-converting combinations of nanocrystal emitters for warm-white light generation with high color rendering index," Appl. Phys. Lett., vol. 92, pp. 031102-1-031102-3, 2008.

[20] H. S. Chen, D. M. Yeh, C. F. Lu, C. F. Huang, W. Y. Shiao, J. J. Huang, C C. Yang, I. S. Liu, and W. F. Su, "White light generation with CdSe-ZnS nanocrystals coated on an InGaN-GaN quantum-well blue/green twowavelength light-emitting diode," IEEE Photon. Technol. Lett., vol. 18 , no. 13, pp. 1430-1432, Jul. 2006.

[21] H. Chen, C. Hsu, and H. Hong, "InGaN-CdSe-ZnSe quantum dots white LEDs," IEEE Photon. Technol. Lett., vol. 18, pp. 193-195, Jan. 2006.

[22] H. V. Demir, S. Nizamoglu, T. Ozel, E. Mutlugun, I. O. Huyal, E. Sari, E. Holder, and N. Tian, "White light generation tuned by dual hybridization of nanocrystals and conjugated polymers," New J. Phys., vol. 9, p. 362,2007

[23] S. Nizamoglu, T. Ozel, E. Sari, and H. V. Demir, "White light generation using $\mathrm{CdSe} / \mathrm{ZnS}$ core-shell nanocrystals hybridized with InGaN/GaN light emitting diodes," Nanotechnology, vol. 18, pp. 065709-1-065709-5, 2007.

[24] S. Nizamoglu and H. V. Demir, "Hybrid white light sources based on layer-by-layer assembly of nanocrystals on near-UV emitting diodes," Nanotechnology, vol. 18, pp. 405702-1-405702-4, 2007.

[25] T. A. Klar, T. Franzl, A. L. Rogach, and J. Feldmann, "Super efficient exciton funneling in layer-by-layer semiconductor nanocrystal structures," Adv. Mater., vol. 17, pp. 769-773, 2005.

[26] S. Nizamoglu and H. V. Demir, "Resonant nonradiative energy transfer in $\mathrm{CdSe} / \mathrm{ZnS}$ core/shell nanocrystal solids enhances hybrid white light emitting diodes," Opt. Exp., vol. 16, no. 18, pp. 13961-13968, 2008.

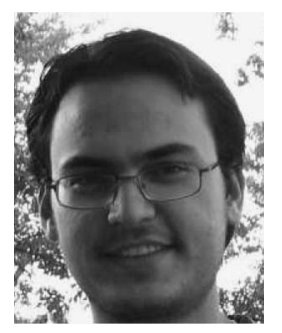

Sedat Nizamoglu (S'09) received the B.S. and M.S. degrees in electrical and electronics engineering and physics in 2005 and 2007, respectively, from Bilkent University, Ankara, Turkey, where he is currently working toward the Ph.D. degree in electrical and electronics engineering.

His current research interests include nanocrystal hybridized novel light-emitting diodes, light-matter interaction of quantum dots, and nanooptics. He has authored or coauthored more than 40 journal and conference papers. His work on "White light generation using $\mathrm{CdSe} / \mathrm{ZnS}$ core-shell nanocrystals hybridized with InGaN/GaN light emitting diodes" is highlighted as a featured article on the hard cover of the journal Nanotechnology by Institute of Physics (vol. 18, no. 6 in the issue of February 14, 2007).

Mr. Nizamoglu is the recipient of Bilkent University Graduate Scholarship (2005-present) and the National Scholarship by the Scientific and Technological Research Council of Turkey (2006-2007). He is a Student Member of the Optical Society of America (OSA) and the International Society for Optical Engineering (SPIE).

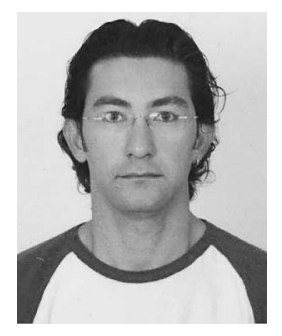

Emre Sari (S'02) received the B.S. and M.S. degrees in electrical and electronics engineering in 2005 and 2007, respectively, from Bilkent University, Ankara, Turkey, where he is currently working toward the Ph.D. degree in electrical and electronics engineering.

His current research interests include the development of novel blue and UV quantum modulators and high-brightness light-emitting diodes.

Mr. Sari is the recipient of Bilkent University Undergraduate Scholarship (2001-2005) and the National Graduate Fellowship by the Scientific and Technological Research Council of Turkey both for his M.S. and Ph.D. programs (2006-2007 and 2008present). He is a Student Member of the IEEE Lasers and Electro-Optics Society (LEOS). 


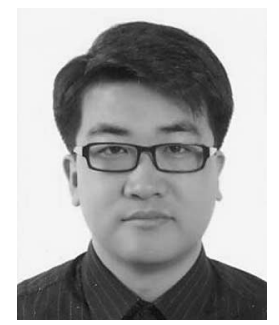

Jong-Hyeob Baek received the B.S., M.S., and Ph.D. degrees in materials science and engineering from Korea University, Seoul, Korea, in 1989, 1991, and 1999, respectively.

He was a Senior Research Staff at the Electronics and Telecommunications Research Institute (ETRI), where he was engaged in research on the epitaxial growth of vertical cavity surface-emitting lasers (VCSELs) from 1991 to 1999 . He was a Postdoctoral Fellow at the University of California, San Diego, and a Member of the Technical Staff at Nova Crystal, Inc. In October 2002, he joined Korea Photonics Technology Institute (KOPTI), Gwangju, Korea, where he was engaged in research on the advanced epi/chip technologies of high-efficiency, high-power R/G/B/UV LEDs, and is currently responsible for developing $380 \mathrm{~nm}$ UV LEDs and GaN/Si blue LEDs. He is also engaged in research on nanotechnology-based fabrication process, wafer level packaging process, and high electrostatic discharge (ESD) electrode design. His research is multidisciplinary and involves close collaboration with academic and industrial groups.

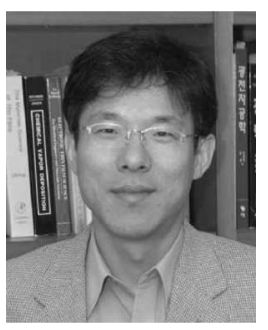

In-Hwan Lee received the B.S., M.S., and Ph.D. degrees in materials science and engineering from Korea University, Seoul, Korea, in 1991, 1993, and 1997, respectively.

During 1997-1999, he was a Postdoctoral Fellow at the Northwestern University. He joined Samsung Advanced Institute of Technology, where he was an Epitaxial Team Leader and developed a world-topclass high-power $[50 \mathrm{~mW}$, continuous wave $(\mathrm{CW})]$ $\mathrm{InGaN} / \mathrm{GaN}$ violet laser diode for optical storage source (Blu-ray). Since 2002, he has been a faculty member in the School of Advanced Materials Engineering, Chonbuk National University, Chonju, Korea. His current research interests include metal-organic chemical vapor deposition (MOCVD) growth and characterizations of GaNbased optoelectronic materials and devices, and transparent electronics based on $\mathrm{ZnO}$ thin films. He is the author or coauthor of about 100 scientific papers in referred journals. With the sabbatical research grant from LG foundation, he is now on leave to Yale University, New Haven, CT (2008-2009).

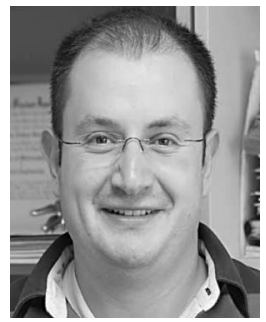

Hilmi Volkan Demir (S'97-M'04) received the B.S. degree in electrical and electronics engineering from Bilkent University, Ankara, Turkey, in 1998, and the M.S. and Ph.D. degrees in electrical engineering from Stanford University, Stanford, CA, in 2000 and 2004, respectively. He received the Docent title (Associate Professor) in optics and photonics from Turkish Council of Higher Education in 2007.

Since 2004, he has been a faculty member at Bilkent University, with joint appointments at the Department of Electrical and Electronics Engineering and the Department of Physics, where he is the Founding Director of the Devices and Sensors Laboratory and the Associate Director of the Nanotechnology Research Center. His current research interests include the development of nanocrystal embedded novel optoelectronic devices (including solid-state lighting devices, photovoltaic devices, modulators, and switches), innovative optoelectronic devices and sensors, light-matter interaction at nanoscale, and nanophotonics. He has authored or coauthored over 100 peer-reviewed research articles in major scientific journals and conferences, presented over 100 invited seminars and talks around the world, and holds over ten patents at various stages of the process.

Dr. Demir is the recipient of 2008 Orta Dogu Teknik Universitesi (ODTU) Prof. M. N. Parlar Young Scientist Award, the 2007 European Science Foundation European Young Investigator (ESF-EURYI) Award, the 2006 Turkish National Academy of Sciences Distinguished Young Scientist Award (TUBAGEBIP), and the 2005 European Union Marie Curie Fellowship. 